de l'action; d'observer sans passion, de discuter les espèces si var'iées et les manières dont elles peuvent varier; de metre en main de l'industriel tous les moyens qu'il peut souhater avoir pour réaliser les variations utiles. En un mot suivre la nature pour la diriger.

Commandant AUDEBRAND, Ingénieur,

ancien elèv de l'Ecole Polytechneque.

\section{Essai de Recensement provisoire des prineipales Forces hydrauliques}

Conmmunication de M. DF LA Brossp à l'Associalıon Francaise pour luvancenent des Scicnces. - Congrès de Grenoble 1904.

on ne pourra évaluer avec quelque exactitude liimportance des forces hydrauliques que lorsque des jaugeages, nombreux et prolongés, auront donné une connaissance à jeu près juste du régime de nos cours d'eau et lorsque leurs rives auront été dotées de bons nivellements avec repères d'altitude.

Cependant, et sans attendre jusque-là, il peut sembler désirabie de tenter au moins un aperçu de l'importance de ces forces, de chercher a savoir par exemple si les Alpes nous réservent quelques centaines de mille ou plusieurs millions de chevaux, car les essais de recensernent faits jusqu'ici n'ont donné que des renseignements assez vagues à cet érard.

J'ai cherché, à défaut de mieux, dans les données statistiques des services hydrauliques des éléments qui permissent une évaluation provisoire au moins grossière de ces ressources, et peut-être ne sera-t-il pas saus intérêt d'en donner ici un premier apercu pour la partie septentrionale des Alpes comprenant les départements de la Haute-Savoie, de la Savoie, de l'Isère et des Hautes-Alpes.

Un recensement sommaire a déjà eu lieu d'ailleurs pour la Savole et les Hautes-Alpes par les soins des services locaux (1).

Il faut reconnaître que les débits indiqués par les statisti ques anciennes, notamment par les tableaux dressés dans chaque département en exécution des circulaires ministérielles des 30 juillet 1861 et 4 juillet 1878 , sont en général inexacts. Les minima y sont souvent exagérés par suite de linsuffisance d'observations précises sur les basses eaux. Il est donc impossible de tirer de ces statistiques des résullats un peu sérieux. Aussi a-t-on dû recourir pour corriger les plus grosses erreurs à des renseigruements directs pris auprès de certains industriels et des services locaux. D'ailleurs, ces renseignements eux-mêmes ne sont pas donnés comme certains, puisqu'il n'y a encore presque nulle part de bons jaugeages, mais ils rectifient du moins les erreurs les plus grossières des statistiques anciennes et ils peuvent, en attendant mieux, donner une idée plus ou moins approchée des débits de certains cours d'eau.

Il s'agit donc d'une approximation imparfaite : il importe de ne pas le perdre de vue. Toutefois, telle que nous sommes en mesure de l'établir et sous les réserves que l'on vient de dire, cette approximation peut déjà donner une idee de l'ordre de grandeur de nos puissances hydrauliques, en attendant que les jaugeagres méthodiques en voie d'organisation apportent dans ces évaluations un peu plus d'exactitude.

Le département de la Haute-Savoie verse ses eaux dans le lacléman et dans le Rhône par les bassins du Trient (Suisse), des Dranses, des Usses, du Fier et de l'Arve. Une

(1) Pour le département de la Savoie l'évaluation a été fatte par $M$. Ingénieur en chef Gotteland, en 1901.

Pour celu des Hautes Alpes, vorr les évaluntions de M. R. Tavernier

at la carte dressce par ses soins pour l'exposition universelle de 1900. petite portion de ce département est tributaire du bassin de l'Isère par le cours supérieur de l'Arly. Le recensement provisoire, effectué avec les données plus ou moins imparfaites que l'on possède actuellement, donnerait pour la Haute-Savoie un total d'au moins 100000 chevaux (2) d'étiage et 375000 chevaux en eaux moyennes. J'ai de bonnes raisons pour croire que les nouveaux jaugeages plus précis augmenteront très notablement ces chiffres.

Lè département de la Savoie verse ses eaux dans le Rhône et dans l'Isère par les bassins du Haut-Fier (Chéran), du lac du Bourget, du Flon, du Guiers, de l'Arly et de l'Arc. M. l'Ingénieur en chef Gotteland a fait, sur des bases un peu différentes des miennes, mais qui s'en rapprochent néanmoins sutfisamment, un recensement des forces correspondantes qu'il a évaluées (en chiffres ronds) à 320000 HP d'étiage et $650000 \mathrm{HP}$ en eaux moyennes.

Le département de l'isère verse ses eaux dans le Rhône et l'Isère par les bassins du Guiers, de l'Hières, de la Boulbre, du Bréda, du Drac, de la Bourne, etc... Le recensement provisoire lui attribuerait environ $350000 \mathrm{HP}$ à l'étiage et $800000 \mathrm{HP}$ en eaux moyennes.

Pour les Hautes-Alpes, M. Tavernier avait trouvé environ 300000 et 500000 chevaux. Les renseignements relatifs aux autres départements du sud-est ne sont pas encore assez nombreux pour étendre davantage cet aperç. Quoi qu'il en soit, cette évaluation provisoire donnerait pour les quatre départements de la Haute-Savoie, de la Savoie, de I'Isère el des Hautes-Alpes, un total de 1 million de chevaux d'étiage, savoir :

\begin{tabular}{|c|c|c|c|}
\hline \multirow{2}{*}{ DÉPARTEMENTS } & \multirow{2}{*}{ SUPERFICIE } & \multicolumn{2}{|c|}{$\begin{array}{l}\text { RECENSEMENT PROVISOIRE } \\
\text { DES FORCES MDUALLOULS }\end{array}$} \\
\hline & & d'eitiage & en eaux moyennes \\
\hline Haute-Savoie (*). & 459.801 & 100.000 & 375.000 \\
\hline Savole.. & 618.791 & 320.000 & 650.000 \\
\hline Isère. & 823.658 & 350.000 & 800.000 \\
\hline Haules-Alpes. & 564.311 & 300.000 & 500.000 \\
\hline $\begin{array}{l}\text { Ensemble pour les } 4 \text { dépar- } \\
\text { tements considérés.... }\end{array}$ & 2.466 .561 & 1.070 .000 & 2.325 .000 \\
\hline Solt en chiffres ronds. & & 1.000 .000 & 2.300 .1300 \\
\hline
\end{tabular}

${ }^{*}$ ) Chiffes vraisemblablement insuffisants.

Cette statistique sommaire est évidemment bien imparfaite. Ce n'est point par département qu'il y aurait lieu de proceder logiquement, mais par bassins. Toutefois ces chiffres n'ayant aucune prétention de rigueur et devant fournir simplement un aperçu de l'ordre de grandeur de nos ressources hydrauliques, il sera peut-être plus facile de les retenir sous cette forme. J'ai d'ailleurs tout lieu de penser qu'ils représentent des minima et que les études ultérieures conduiront à les majorer notablement. Dès maintenant, il semble que les quatre départements qui nous servent en ce moment d'exemple recèlent une puissance hydraulique d'au moins un million de chevaux aux plus basses eaux d'étiage et qui se maintient pendant six mois de l'année entre deux millions et deux millions et demi de chevaux, c'est-à-dire à près de 1 cheval par hectare de superficie moyenne. Ce sont là des chiffres dignes d'attention, surtout si l'on considère que, dans cette évaluation, n'est pas compris le Rhône qui constitue d'autre part, à lui seul, dans la même région, un important réservoir d'énergie.

(2) Nous prendrons toujours pour base de nos évaluations le poncelet loo hiloummes par seconde équivalent, comme on le sait, au cheval (100 kilogrammes par seconde) equivalent, comme on quand on suppose à ceux-ci un rendement de $75 \%$. Cotte unité tend de plus en plus a se rénéraliser dans' toutes les branches des industries hydrauliques. Elle se marque par la notation HP. 
Ces quatre départements représentent là peu près $4,5 \%$ de la superficie totale de la France; sans doute ils sont proportionnellement les mieux pourvus en forces hydrauliques grâce à leur relief considérable et à l'abondance de leurs eaux; cependant, les autres départements du sud-est jusqu'au littoral méditerranéen ont aussi de puissantes réserves dans les bassins du Var et de la Durance; la région des Pyrénées, le Plateau Central, les Vosges et le Jura en possèdent également.

Si l'onvoulait tenter sur ces bases, malgré leur imperfection, un essai de recensement général, on pourrait réunir les divers départements en quatre groupes principaux:

Le premier groupe, comprenant les quatre départements cités plus haut, embrasse 24666 kilomètres carrés et compte, comme on vient de le dire, environ un million de chevaux à l'étiage ;

Le second groupe comprenant les autres départements du sud-est depuis la Dróme jusqu'aux Alpes-Maritimes et les six départements de la bordure pyrénéenne embrasse une superficie de 63166 kilomètres carrés. En l'affectant par rapport au premier groupe du coefficient $1 / 2$ on lui attribuerait 1300000 chevaux.

Le troisième groupe comprenant 12 départements du Centre et 6 de l'Est embrasse 91000 kilomètres carrês; avec le coefficient $1 / 4$, il aurait 900000 chevaux;

Enfin le surplus du territoire formant le quatrième groupe, avec 357000 kilomètres carrés et le coefficient $1 / 10$ aurait 1400000 chevaux, ce qui donnerait pour la France entière un minimum de 4 millions et demi de chevaux d'étiage.

Mais on sait depuis longtemps que la richesse hydraulique d'un pays ne se mesure pas seulement à son minimum d'étiage et de nombreuses applicalions industrielles nous apprennent que l'on peut utilement profiter des eaux surabondantes des saisons favorables, représentées tout au moins par le régime moyen. C'est donc à 9 ou 10 millions de chevaux qu'il faut évaluer la richesse hidraulique efficace de notre territoire. Or c'est précisément la puissance totale nominale des machines à vapeur qui, d'après les dernières statistiques officielles de l'industrie minérale, se décompose ainsi :

Etablissements industriels et agricoles...... 1900000 chevaux.

Chemins de fer et tramways............ 6200000

Batellerie et navigation fluviale .......... 80000

Ensemble.....

Ainsi la puissance hydraulique de nos cours d'eau ne serait pas moindre que celle de tout notre outillage à vapeur. En réalité elle peut satisfaire un ensemble de besoins bien supérieur parce que l'utilisation en est beaucoup plus complète dans le temps.

Les statistiques de machines à vapeur comprennent en effet pour leur force nominale une foule dappareils à marche discontinue, notamment pour les chemins de fer dont les locomotives, machines de secours et de rêserve, etc.... ne donnent leur pleine puissance qu'un très petit nombre d'heures par jour. Il en est de même dans beaucoup d'industries dont les moteurs thermiques font face à un service horaire discontinu.

Au contraire, les forces hydrauliques permettent d'assurer des services permanents de 24 heures par jour, toute l'année, pour les forces d'étiage, six mois par an pour les forces moyennes, ce qui leur donne un intérêt tout spécial, facile a faire ressortir par la considération des heures'dé service annuel.

Si l'on admet par exemple que les machines à vapeur recensées par les statistiques administratives travaillent en moyenne, savoir :

(A) Dans les établissements industriels et agricoles, 18 heures par jour;

(B) Dans les chemins de fer et tramways, 8 heules par jour;
(C) Dans la batellerie et navigation fiuviale, 12 heures par jour.

On obtient pour l'année entière:

$$
\begin{aligned}
& \text { (A) } \ldots \ldots \ldots \ldots 18^{\mathrm{h}} \times 365 \mathrm{j} \times 1^{\mathrm{HP}}, 9 \times 10^{6}=12312 \\
& \text { (B) } \left.\ldots \ldots \ldots \ldots \quad 8 \times 365 \times 6 \quad 2 \times 10^{6}=18104\right\} \times 10^{6} \\
& \text { (C) } \ldots \ldots \ldots 12 \times 360 \times 8 \quad 08 \times 10^{6}=346 \\
& \text { Ensemble.......... } 30762 \times 10^{\circ}
\end{aligned}
$$

Soit, en chiffre rond, 30.000 millions ou 30 milliards de chevaux-heures dans l'année.

Or l'aménagement intégral de nos forces hydrauliques, d'après les évaluations ci-dessus procurerait :

$$
\begin{aligned}
& 1^{\circ} \text { A l'étiage..., } 24^{\mathrm{h}} \times 365 \mathrm{j} \times 4^{\mathrm{H}}, 5 \times 10^{6}=39420 \\
& \text { 20 Eaux moyennes } \left.24 \times 180 \times 5 \times 10^{6}=21600\right\} \times 10^{\circ} \\
& \text { Ensemble.......... } 61020 \times 106
\end{aligned}
$$

Soit, en chiffre rond, 60.000 millions ou 60 milliards de chevaux-heures dans l'année, c'est-à-dire le double de ce que procure actuellement l'outillage à vapeur.

Sans doute, ces évaluations présentent une base contestable et reposent sur des données encore incertaines. Il n'en demeure pas moins évident que notre territoire possède dans son appareil hydraulique un admirable réservoir d'énergie dont la mise en valeur bien conduite augmentera dans une énorme proportion la richesse générale.

Je m'en tiendrai pour le moment à cette réconfortante constatation dont il m'a semblé utile de faire part à l'Association française pour l'avancement des Sciences au moment. ou, pour la seconde fois, elle vient tenir sa session au pays; qui fut le berceau de la houille blanche et qui demeure le: principal théâtre de cette industrie.

R. DE LA BRosse. Ingénieur en chef des Ponts et Chaussées.

\section{RemaRque RELATIVE a LA STATISTIQUE PRÉCÉDENTE}

A côlé du charbon brûlé par l'outillage $\dot{a}$ vapeur, il y a lieu de considérer - ce que l'on ne fait pas souvent - le combustıble employé dans les usines métallurgiques, de produits chimiques, d'éclairage par le gaz, les verreries, les chaufourneries, elc., etc... Cette consommation est considérable et il serait instructıf de comparer son importance à celle de la première. $0 r_{\text {r }}$ la " houlle blanche ", comme nous le savons tous ici, n'a pas. seulement son emploi dans les moteurs, mais encore el surtout dans les fours électriques et les appareils de production électrochimique, qui se substituent peu à peu aux engins de la vieille industrie. Or, si nos forces hydrauliques peuvent nous fournir le double de ce que procure l'outillage da vapeur, sont. elles cependant suffisantes pour remplacer, le cas échéant, tout le charbon brúlé dans nos hauts-lourneaux, nos fonderies, nos usines de produits chımiques, céramıques, etc.? Il semblerait. bien que oul, d'après le très intéressant travail de M. DE LA BRosse.

L'énergie de nos forces hydrauliques peut être transportée électriquement à très grande distance des lieux de génération; mais le transport de cette énergie s'arrête aux points où celle produile par la houille noire revient meilleur marché. Les posi:tions respectives de nos mines, de nos centres de ravitanllement en charbon el de nos chutes d'eaux sont telles qu'en un grand nombre de points de notre territoire, la force motrice à vapeur resteŕa longlemps encore meille:ır marché que l'énergie hydro-électrique, par conséquent, tout le travail mócanique, actuellement du à la combustion du charbon ne pourra sans doute jamais être remplacé par nos forces hydrau'ıques : la plupart des industries mécaniques ne peuvent se déplacer et ld houille blanche ne: pourra pas aller assez économlquement jusqu'à elles. Armettons donc que nos forces hydraılıques remplacent seulement Ja moitié - ce qui est déjá beaucoup - de notre outillage à vapeup et sur ses 30 milhards de chevaux-heure lui en fournissent 15. Il restera a la disposition de nos forges, fonderies, usines de produits chimiques, verreries et aulres usines de même espëce: $60-15=45$ milliards de chevaux-heure. 
Ces usines, où le four alimenlé par la houille est l'organe vital, vont se déplacer le jour où elles trouveront de l'énergie calorifique à meilleur marché et se transporter au pied des chutes, quand la science et le progrès économique leur procureront l'énergie hydro-électrique à meilleur compte que l'effet calorifique dû l'emploi de la houille noire. Or, ces 45 milliards de chevauxheures, correspondent-ils à ce que peut donner le charbon brûlé par elles?

on sait que 1 cheval-heure correspond à : $3.600 \times \frac{75}{425}$ calories. La puissance qui nous occupe représente donc :

$$
3.600 \times \frac{75}{425} \times 45 \times 10^{9}=28.588 .000 .000 .000 \text { calories }
$$

La combustion de 1 kılogramme de houille moyenne qualité donne 7.000 calories; mais, étanl donné le faible rendement des fours et appareils de chauffage en général, on peut admettre pour fixer les idées - et non pour faire un calcul rigoureux - que la fusion, cuisson ou réaction qui nécessite 10.100 calories dans l'un de ces appareils, exige la combustion de $10 \mathrm{kilogs}$ de houille au moins. Le nombre de calories trouvé précédemment correspondratt à la combustion de :

$\frac{28588 \times 10^{9}}{10^{3}}=28$ à 29 millions de tonnes de charbons.

Mettons 25 millions pour tenir compte aussı du rendement des appareils électrothermiques. C'est plus de la moitié de ce que nous brûlons annuellement en France dans l'ensemble des machines et appareils de toutes nos industries; mécanique, métallurgie, transports, chauffage, chimie, gaz, etc.

Il semblerait donc à peu près certain que nous avons assez de forces hydrauliques pour remplacer le charbon dans toutes les branches de nos industries que les progrès de la science mettront ì mème de pouvoir économiquement s'alimenter en houille blanohe. Les conclusions du travail de M. DE LA BRossE concordent d'ailleurs parfaitement avec celles que M. le commandant AudeBRAND a fait ressortir dans son étude sur la même question " La Houille blanche en France $\triangleright$, parue dans les Annales de Géographie, no de janvier 1904.

E.-F. COTE.

\section{ÉTUDE SUR LES}

\section{BARRAGES EN MHÇONNERIE}

\author{
et Murs de Réservoirs
}

(suite)

Profil triangulaire. - La méthode de M. Maurice Lévy, appliquée au triangle à parement amont vertical, donne :

$$
\begin{array}{ll}
P=\left(K-\frac{1}{\operatorname{tg}^{2} \beta}\right) y & Q=\left(\frac{2}{\operatorname{tg}^{2} \beta}-K\right) \frac{1}{\operatorname{tg} \beta} \\
P^{\prime}=K-\frac{1}{\operatorname{tg}^{2} \beta} & Q^{\prime}=0 \\
P^{\prime \prime}=0 & Q^{\prime \prime}=0
\end{array}
$$

Il en résulte pour $n, t$ et $n_{1}$, les expressions suivantes :

$$
\begin{gathered}
n=\left(K-\frac{1}{\operatorname{tg}^{2} \beta}\right) y+\left(\frac{2}{\operatorname{tg}^{2} \beta}-K\right) \frac{x}{\operatorname{tg} \beta} \\
t=\frac{x}{\operatorname{tg}^{2} \beta} \quad n_{1}=y
\end{gathered}
$$

La condition de non soufflure au parement aval, $\left(n_{1} \geqslant 0\right)$, est toujours satisfaite puisque $n_{1}$, est toujours égal à $y$ quel que soit l'angle au sommet $\beta$.

La composante tangentielle $t$ de la pression totale sur un élément horizontal, n'est pas autre chose que la composante de la poussée $F$, au point considéré d'abscisse $x$. Cette composante $t$ croit proportionnellement à $x$ : nulle au parement amont, elle est maxima au parement aval et y a pour valeur :

$$
t=\frac{e}{\operatorname{tg}^{2} \beta}=\frac{y}{\operatorname{tg} \beta}=n^{\prime \prime} \operatorname{tg} \beta
$$

Si l'on fait la somme des forces $t$ réparties sur le joint on retrouve bien la poussée $F$ :

$$
\int_{0}^{e} \frac{x \mathrm{~d} x}{\operatorname{tg}^{2} \beta}=\frac{e^{2}}{2 \operatorname{tg}^{2} \beta}=\frac{y^{2}}{2}=F
$$

Si l'on calcule le rapport $\frac{t}{n}$, qui n'est pas autre chose que la valeur de la tangente de langle que fait la composante normale $n$ avec la pression totale exercée en un point d'un élément horizontal, on trouve que ce rapport, nul au parement amont, croit avec $x$ et est précisément égal à $\operatorname{tg} \beta$ sur le parement aval, ce qui confirme que la pression totale au parement aval est bien parallèle à ce parement.

Si l'on applique le raisonnement de M. Bouvier, non plus à la résultante $R$ des forces appliquées au mur au-dessus d'un joint, mais bien à la pression totale s'exerçant en chaque point, on voit que la méthode Bouvier, ainsi modifiée, conduit au même résultat que la méthode Lêvy, puisque sur le parement aval on a $\operatorname{tg} \alpha=\operatorname{tg} \beta$.

La compression maxima $A$ a pour expression :

$$
A=\frac{1}{2}\left\{\begin{array}{c}
\left(K+1-\frac{1}{\operatorname{tg}^{9} \beta}\right) y+\left(\frac{2}{\operatorname{tg}^{2} \beta}-K\right) \frac{x}{\operatorname{tg} \beta}+ \\
\sqrt{\left[\left(K-1-\frac{1}{\operatorname{tg}^{2} \beta}\right) y+\left(\frac{2}{\operatorname{tg}^{2} \beta}-K\right) \frac{x}{\operatorname{tg} \beta}\right]^{2}+4 \frac{x^{2}}{\operatorname{tg}^{4} \beta}}
\end{array}\right\}
$$

qu'on peut mettre sous la forme :

$$
A=\frac{\lambda+\theta x}{2}+\frac{1}{2} \sqrt{\left(\lambda^{1}+\theta x\right)^{2}+\gamma^{2} x^{2}}
$$

Si $\theta>0$, la fonction $A$ est croissante et est par suite maxima sur le parement aval. Ceci a lieu lorsque $\operatorname{tg}^{2} \beta<\frac{2}{\bar{K}}$. Or, avec les maçonneries, on a toujours $K>\frac{1}{K-1}$, et comme lon cherche toujours à réaliser le minimum de maçonneries, $\operatorname{tg}^{2} \beta$ sera toujours dans le voisinage de $\frac{1}{K-1}$ et $A$ sera pratiquement toujours maximum au parement aval.

Si l'on avait $\theta<0$, ce qui serait le cas de profils exagérés, $A$ serait d'abord décroissant, pour croitre ensuite, et, si le profil était tout à fait exagéré, il pourrait arriver que la fonction $A$ fut continuellement décroissante, le maximum de $A$ se trouvant alors sur le parement amont. Lorsque $\operatorname{tg}^{2} \beta$ devient très grand, la valeur maxima de $A_{0}$ tend vers la valeur $K y$, qui est précisément la valeur de la pression à vide sur le parement amont. Ce résultat pouvait se prévoir a priori, car alors la poussée devient négligeable devant le poids de la maçonnerie.

$\mathrm{Au}$ fur et à mesure que le réservoir se vide, le profil devient de plus en plus exagéré et la valeur de $A$ cesse d'être maxima au parement aval pour le devenir sur le parement amont.

Sur le parement amont, $x=0$ et $t=0$ de sorte que l'on a, tant que $n^{\prime}>y$, c'est-à-dire $\lambda^{\prime} \geqslant 0$.

$$
A_{0}=n_{0}=n^{\prime}=\left(K-\frac{1}{\operatorname{tg}^{2} \beta}\right) y
$$

Lorsque $\lambda^{\prime}<0$, le radical tend vers $\lambda^{\prime}$, compté en valeur 